\title{
Depth measurement of closely spaced surface cracks by the electric potential method
}

\author{
Petr Shkatov ${ }^{1}$, Irina Lisitsyna ${ }^{1}$, Anna Sandulyak $^{1, *}$ and Hasan Bulut ${ }^{2}$ \\ ${ }^{1}$ Moscow technological university, Moscow, RF, \\ ${ }^{2}$ Department of Mathematics, University of Firat, Elazig, Turkey
}

\begin{abstract}
The study results are provided for the electropotential converter signals interacting with two closely spaced parallel surface cracks. The measurement scheme was studied with transducer electrodes placed at the vertices of the rectangle. Study results are important for depth measuring of the surface cracks of stress-corrosion origin.
\end{abstract}

\section{Introduction}

The depth of surface cracks refers to one of the most significant parameters that determines the technical condition of the metal objects of responsible use. To measure the depth of single cracks, the electropotential method has been successfully applied [1, p. 498-502]. This method is based on current passage through the defective area and recording voltage between the potential electrodes. The crack depth is evaluated by the relative change in voltage during transition from the defect-free area (voltage $U_{0}$ ) to the identical defective area (voltage $U_{r}$ ). The value $U^{*}=\left(U_{r}-U_{0}\right) / U_{0}$ depend on metal specific electric conductivity. To exclude the metal magnetic permeability effect on measurement result, the transmitted current is chosen to be constant [2]. In addition to the measured value - crack depth $\mathrm{h}$, $U^{*}$ value is also affected by such parameters as crack length $\ell$, thickness $\mathrm{T}$ of the controlled section, surface curvature, crack plane deviation from the plane orthogonal to the surface of the controlled object. The influence of these factors and methods for measuring the crack depth $\mathrm{h}$ with the appropriate adjustment are considered in [3-6].

Known studies refer to measuring the depth of single cracks. At the same time, in practice, it is often necessary to measure the depth of surface cracks that form a network on the surface of a controlled area. This problem is typical for zones with cracks of stresscorrosion origin.

\section{Selection of electropotential converter}

When measuring the depth of surface cracks, the current and potential electrodes of the electropotential converter (EPC), usually [1,2], are set along a single line (Fig. 1) perpendicular to the plane of the crack. Hereafter, we will call similar EPC as linear for short. It is not possible to apply linear EPCs in measurements within the crack network zone if the distance between adjacent cracks is comparable to the cracks depth. This is due to the fact that to ensure an acceptable measurement error, the distance between the current electrodes should be several times greater than the measured depth of the crack [3]. However, in this case there will be more than one crack between the current electrodes which makes the measurements impossible. Due to this limitation the measurements using electropotential method in the cracks network zone are not applied in practice.

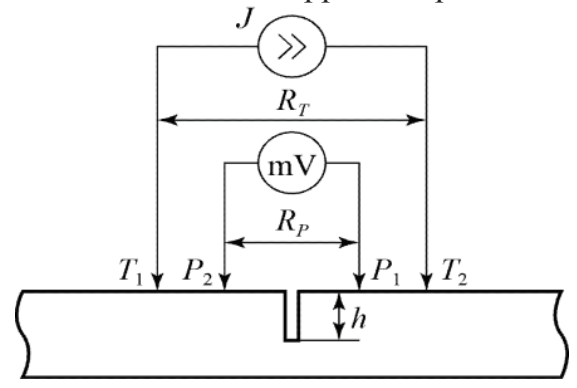

Fig. 1. Electropotential converter with placement of electrodes along the line ("linear" EPC).

To address this issue it is advisable to use the arrangement of the electrodes at the corners of the rectangle (Fig. 2). Hereafter, we will call for short the similar electropotential converters as rectangular. The use is known of the rectangular EPC for measuring the specific electric conductivity $[2,11]$, creep of metals in the welded joints zone [7] and depth of single cracks [810]. EPC advantages include a large relative sensitivity, which is important, for example, in the measurement of cracks of shallow depth [8], as well as the possibility of placing electrodes in areas with a cylindrical surface shape [9].

\footnotetext{
Corresponding author: anna.sandulyak@mail.ru
} 


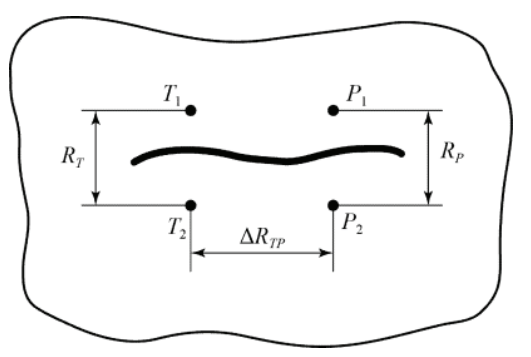

Fig. 2. Electropotential converter with placement of electrodes at the corners of the rectangle ("rectangular" EPC).

The rectangular EPC advantages with respect to the measurement of cracks network zone is that the pairs of current and potential electrodes can shift relative to each other without approaching adjacent cracks. In this case, as shown in [9-10], the range of possible measurement of the cracks depth is changing. In other words, increasing the distance $\triangle$ RTP between pairs of current and potential electrodes for a rectangular EPC (Fig. 2) leads to the same effect as an increase in RT distance for linear EPC (Fig. 1).

\section{Study of the adjacent crack effect on stress introduced by a surface crack with a measured depth}

The performed analysis shows the expediency of using rectangular EPC to measure the depth of surface cracks in the cracks network zone. To determine the possibility of measuring the depth of the surface crack in the cracks network zone by the finite elements method (FEM), the theoretical calculations were carried out in accordance with the geometric design model shown in Fig. 3.

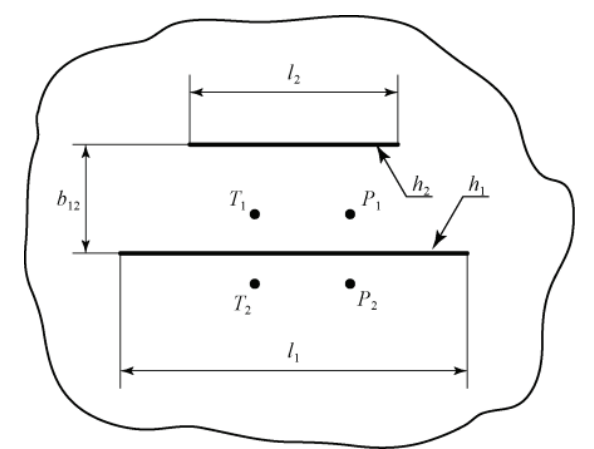

Fig. 3. Geometric design model of interaction of the rectangular EPC with two cracks parallel to each other.

To estimate the effect of an adjacent crack with a depth $h^{*}{ }_{2}$ on signal recorded when placing a rectangular EPC above surface crack with a depth of $h *{ }_{1}$, the calculations were carried out for a variation of the distance $b^{*}{ }_{12}$ between the cracks planes and their depths. As an illustration Fig. 4 shows the voltage dependence $U^{*}{ }_{1}$ EPC, located above the crack with a depth of $h^{*}{ }_{1}$ in a function of depth $h^{*}$ of the adjacent crack for a number of $b^{*}{ }_{12}$ values for $\Delta R^{*}{ }_{T P}=1, \ell 1^{*}=\ell_{2} *=20, T \rightarrow \square$. In Fig. 4 -a the value $h^{*}=0.8$, and in Fig. 4 -b $-h{ }_{1}=2$.

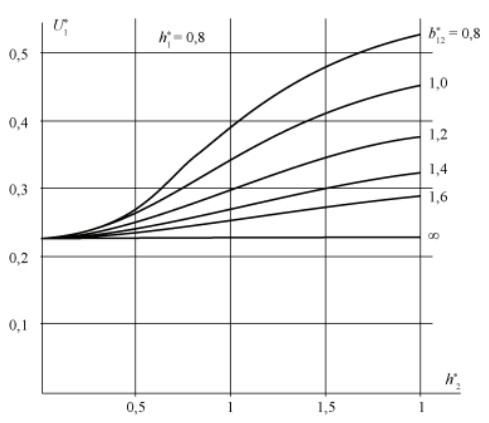

a)

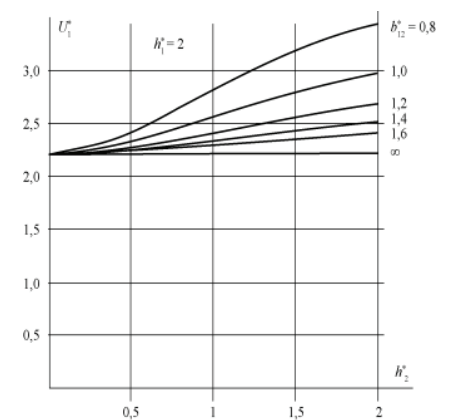

b)

Fig. 4. Dependence $U^{*}{ }_{1}=U^{*}{ }_{l}\left(h^{*}, b^{*}{ }_{12}\right)$ for a rectangular EPC placed above crack with a depth of $h^{*}$ and effect of an adjacent crack with a depth of $h^{*}{ }_{2}$ for $\Delta R^{*}{ }_{T P}=1, \ell_{1}{ }^{*}=\ell_{2}{ }^{*}=20$, $\mathrm{T} \rightarrow \infty: a-a t h_{1}^{*}=0,8, b-$ at $h_{1}^{*}=2$.

It can be seen from these functions that the adjacent crack effect increases monotonically with its increasing depth and decreasing in distance between them. At the same time, with increasing in depth $h^{*}{ }_{1}$ of the main crack, the effect of the adjacent crack increases in absolute value, and decreases on the relative crack.

Calculations show that for distances $b{ }_{12}$ comparable to $\Delta R^{*}{ }_{T P}$ between close-in-depth cracks, the effect of the adjacent crack is large enough. For example, at a value of $b^{*}{ }_{12}=1$ and $h{ }_{1}=h^{*}{ }_{2}=0.8$, the voltage $U^{*}{ }_{1}$ increases by $50 \%$ due to the effect of the adjacent crack. Taking into account the non-linearity of the dependence $U^{*}{ }_{1}=$ $U^{*}{ }_{1}\left(h{ }_{1}\right.$ this will lead to more than $100 \%$ error in measuring the depth $h^{*}{ }_{1}$ of the crack.

Thus, for a reliable measurement of the crack depth in the cracks network zone, it is necessary to take into account the mutual effect of adjacent cracks. This effect depends both on the parameters that are known or can be measured: lengths $\ell_{1}$ and $\ell_{2}$ of each crack, distance $b^{*}{ }_{12}$ between them, metal thickness $T$ in the controlled section, inter-electrode distances, and unknown depths $h{ }_{1}$ and $h{ }_{2}$ of adjacent cracks.

The cracks depth of can be determined by measuring the voltages $U^{*}$ and $U^{*}$ on each of the cracks taking into account all the above-mentioned influencing parameters. This statement can be illustrated by the diagrams shown in Fig. 5-6. 


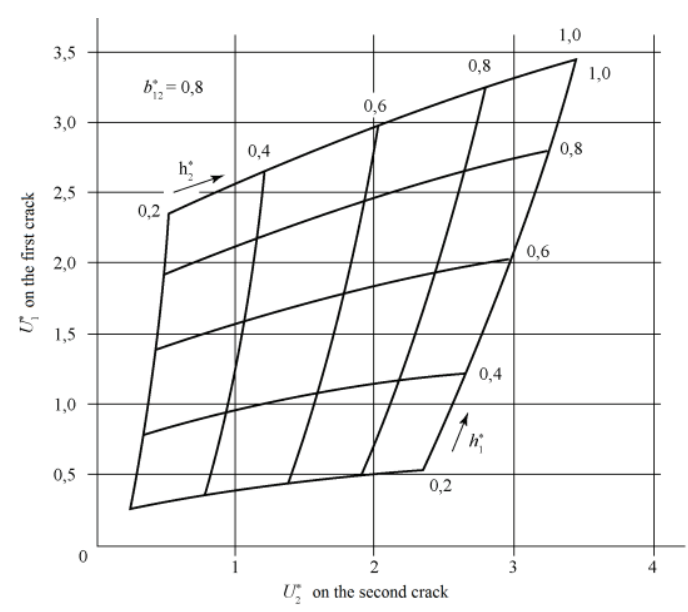

Fig. 5. Functions $U^{*}{ }_{1}=U^{*}{ }_{1}\left(U^{*}{ }_{2}\right)$ for rectangular EPC interacting with two cracks at $b^{*}{ }_{12}=0.8, \Delta R_{T P}^{*}=1, \ell_{1}{ }^{*}=\ell_{2}{ }^{*}=20$, $T \rightarrow \infty$.

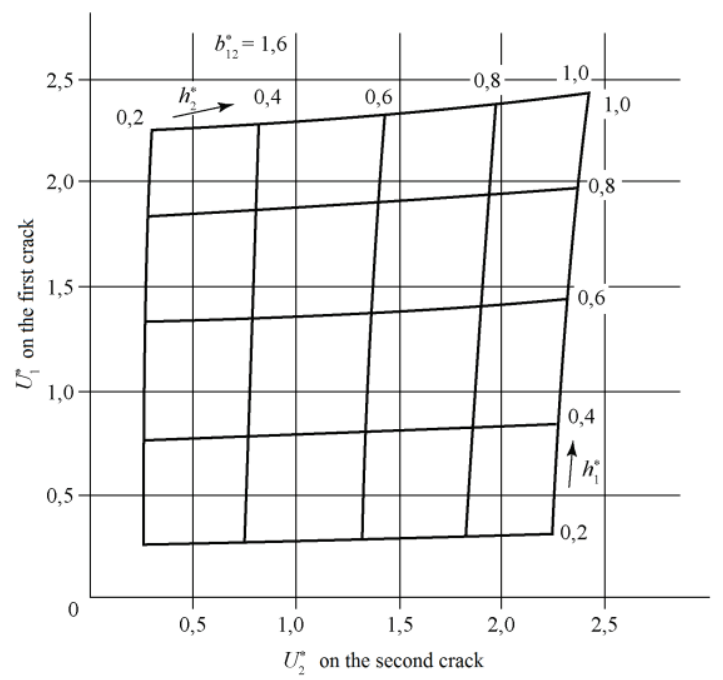

Fig. 6. Functions $U_{1}^{*}=U_{1}^{*}\left(U_{2}^{*}\right)$ for rectangular EEC interacting with two cracks at $b^{*}{ }_{12}=1.6, \Delta R_{T P}^{*}=1, \ell_{1}{ }^{*}=\ell_{2}{ }^{*}=20$, $T \rightarrow \infty$.

They show the measurement results for voltages in $U^{*}{ }_{1}$ and $U^{*}{ }_{2}$ axes for a set of crack depths $h^{*}{ }_{1}$ and $h^{*}{ }_{2}$ with other fixed parameters indicated under the figures. The diagram in Fig. 5 is constructed at a normalized distance $b^{*}{ }_{12}=0.8$ between the cracks, and diagrams in Fig. $6-$ at $b^{*}{ }_{12}=1.6$. The given diagrams show that with increasing distance $b^{*}{ }_{12}$ between cracks, their mutual effect decreases, which leads to the fact that the lines of the diagram become more linear, and their slope relative to the corresponding axis decreases.

\section{Conclusions}

To measure the depth of closely spaced parallel cracks, it is advisable to use an electropotential converter with electrodes placed in the corners of a rectangle (rectangular EEC).

To determine the depth of each of the two cracks effecting each other, it is necessary to measure the input voltage when placing the electropotential converter over each of the cracks, measure the distance $b_{12}$ between them and their lengths $\ell_{1}$ and $\ell_{2}$, and use the previously calculated multidimensional dependencies when interpreting.

Multidimensional dependencies should be calculated for a set of depths $h^{*}{ }_{1}$ and $h^{*}{ }_{2}$, as well as the effecting factors $b_{12}, \ell_{1}$ and $\ell_{2}$, determined by direct measurements.

In a particular and practically important case of measuring the depth of long cracks, the recorded signals, with an acceptable error, can be interpreted using the pre-calculated diagrams $U^{*}{ }_{1}=U^{*}{ }_{1}\left(U^{*}{ }_{2}\right)$ calculated for a number of values of $b^{*}{ }_{12}$.

\section{Acknowledgements}

The research is conducted with financial support from RFFI within the frameworks of research project № 16-38-60034 mol_a_dk.

\section{References}

1. V.P. Vavilov, K.V. Podmasteriev, F.R. Sosnin, et al. Non destructiv control. Under redaction of V.V.Kliuev. Moscow (2006).

2. E.I. Brainin. Control of elements of electrical machines and devices by an electropotential method, Moscow, Energiya (1980).

3. P.N. Shkatov. Research of an error of measurement of depth of cracks by an electropotential method. Proceedings of the $14^{\text {th }}$ Russian science conference «Nondestructive control and diagnostics», Moscow (1996).

4. P.N. Shkatov. Increased preciseness of deep defects defecting through electropotential method. $7^{\text {th }}$ European Conference on NDT, Copenhagen (1998).

5. P.N. Shkatov. The solution of the return problem of an electropotential defektometry for a superficial crack of final length. Proceedings of the $3^{\text {rd }}$ international science conference "Computer methods and the return tasks in nondestructive control and diagnostics", Moscow (2002).

6. P.N. Shkatov. Mech. Eng. 2 (1998).

7. S. Prajapati, P.B. Nagy, P. Cawley. Potential drop detection of creep damage in the vicinity of welds. AIP Conference Proceedings 1430 (2012).

8. G. Gille. NDT, 4 (1971).

9. P.N. Shkatov. A.A. Elisov. Devices, 4 (2013).

10. P.N. Shkatov. I.O. Lisitsyna. Fundamental and applied problems of the equipment and technology, 1, 315 (2016). 